\title{
OPEN Three-dimensional virtual histology in unprocessed resected tissues with photoacoustic remote sensing (PARS) microscopy and optical coherence tomography (OCT)
}

\author{
Benjamin R. Ecclestone ${ }^{1,2}$, Zohreh Hosseinaee ${ }^{1}$, Nima Abbasi ${ }^{1}$, Kevan Bell ${ }^{1,2}$,
} Deepak Dinakaran ${ }^{2,3}$, John R. Mackey ${ }^{2,3}$ \& Parsin Haji Reza ${ }^{1 \bowtie}$

Histological images are critical in the diagnosis and treatment of cancers. Unfortunately, current methods for capturing these microscopy images require resource intensive tissue preparation that may delay diagnosis for days or weeks. To streamline this process, clinicians are limited to assessing small macroscopically representative subsets of tissues. Here, a combined photoacoustic remote sensing (PARS) microscope and swept source optical coherence tomography system designed to circumvent these diagnostic limitations is presented. The proposed multimodal microscope provides label-free three-dimensional depth resolved virtual histology visualizations, capturing nuclear and extranuclear tissue morphology directly on thick unprocessed specimens. The capabilities of the proposed method are demonstrated directly in unprocessed formalin fixed resected tissues. The first images of nuclear contrast in resected human tissues, and the first three-dimensional visualization of subsurface nuclear morphology in resected Rattus tissues, captured with a non-contact photoacoustic system are presented here. Moreover, the proposed system captures the first co-registered OCT and PARS images enabling direct histological assessment of unprocessed tissues. This work represents a vital step towards the development of a rapid histological imaging modality to circumvent the limitations of current histopathology techniques.

Histopathological imaging of tissues is a principal tool in the treatment of cancers. These visualizations provide clinicians with the subcellular tissue characteristics to determine intrinsic disease biology, disease progression, tumor grade, and cancer classification and prognosis. Current histopathological assessment with bright-field transmission microscopy requires tissue preparation with immunohistochemical staining ${ }^{1,2}$. During this workflow, tissues are resected, preserved in formalin, embedded into a paraffin substrate, sectioned with a microtome, and fixed to a microscope slide. Finally, the tissue slides are stained with exogenous dyes such as hematoxylin and eosin (H\&E) to provide contrast ${ }^{1,2}$. Hematoxylin dye reveals cell nuclei, while eosin highlights extranuclear structures of the surrounding tissues ${ }^{2}$. In total, generating these bright-field microscopy compatible preparations takes several days ${ }^{1,2}$. Due to these practical limitations of preparation time and tissue volumes, only a small subset of resected tissue undergoes this histological processing. In the case of breast cancer, less than $\sim 2 \%$ of the excised tissues are assessed with conventional histology ${ }^{3}$. Clinicians must then make diagnostic decisions from the available preparations. Thus, current clinical practices rely heavily on the acuity of the histological workflow. However, each sample preparation step introduces a potential for variability in the resulting tissue preparations. For example, the specimens chosen to undergo histopathology are selected via macroscopic visual inspection and palpation ${ }^{4}$. As a result, the chosen samples may not be representative of the morphology of the bulk specimen and may exclude the most informative tissues. Even within the selected subset of tissues, only limited volumes of nuclei are visualized. Capturing different regions of nuclear and tissue morphology requires repeated thin

${ }^{1}$ PhotoMedicine Labs, Department of Systems Design Engineering, University of Waterloo, 200 University Ave W, Waterloo, ON N2L 3G1, Canada. ${ }^{2}$ illumiSonics Inc., 22 King Street South, Suite 300, Waterloo, ON N2J 1N8, Canada. ${ }^{3}$ Cross Cancer Institute, Department of Oncology, University of Alberta, 116 St \& 85 Ave, Edmonton, AB T6G 2V1, Canada. ${ }^{\varpi}$ email: phajireza@uwaterloo.ca 
sectioning, where each slice captures a $\sim 5 \mu \mathrm{m}$ section of tissue that must be independently fixed to a slide and stained prior to analysis. These compounding challenges have the potential to worsen patient outcomes.

Ideally, three-dimensional (3D) nuclear and extranuclear-tissue morphology could be visualized label-free directly on unprocessed resected samples. This would avoid the delays of tissue preparation, enabling near realtime histological analysis. Moreover, direct imaging of resected tissues would facilitate more thorough analysis of excised specimens, rather than a restricted subset of tissues. This would permit the most highly informative tissues to be submitted, where required, for further immunohistochemical analysis. A technology capable of imaging in this fashion could revolutionize the workflow of anatomic pathology.

Although several novel histological imaging techniques have emerged, no single technology provides labelfree 3D histological imaging of bulk unprocessed resected tissue samples. Most technologies in this histological imaging space require exogenous dyes or tissue clearing to image within specimens. These techniques include microscopy with ultraviolet surface excitation (MUSE) $)^{5}$, fluorescence microscopy ${ }^{6,7}$, non-linear microscopy ${ }^{8,9}$, and light sheet microscopy ${ }^{10,11}$. Objectively, there are few techniques which have achieved measurable success providing label-free histology like contrast in resected tissues. The most notable are stimulated Raman scattering microscopy ${ }^{12,13}$, photoacoustic (PA) microscopy ${ }^{14-19}$ and optical coherence tomography (OCT $)^{20-25}$. Stimulated Raman scattering microscopy has recently provided histological visualizations in resected tissues ${ }^{12,13}$. However, this was applied in a transmission mode architecture only compatible with thin translucent tissue sections ${ }^{12,13}$. Therefore, PA microscopy and OCT are the most promising means to achieve label-free imaging of thick tissue samples.

OCT visualizes endogenous optical scattering contrast using an interferometric detection architecture. The interferometric mechanism enables OCT to capture columns of depth resolved scattering visualizations within tissues in single acquisitions. Combined with spatial scanning, this allows for recovery of 3D volumes of subsurface scattering structures. When applied to histological imaging, OCT has been shown to visualize subsurface features $\sim 2 \mathrm{~mm}$ into tissues ${ }^{20}$. In research settings, OCT has been used to identify and delineate tissue regions, characterize subsurface lesions, and assess biopsy locations ${ }^{20-24}$. While the majority of works in this field focus on macroscopic tissue imaging, some recent publications have proposed ultra-high-resolution OCT systems for assessing submicron scale tissue morphology ${ }^{25}$. Though subcellular visualizations have been achieved, the diagnostic utility of OCT is limited as the optical scattering contrast does not permit sufficient chromophore specificity $^{26}$. As a result, OCT cannot provide direct nuclear contrast within tissues. Without these biomoleculespecific visualizations, OCT cannot match the diagnostic detail provided by the current pathology standard of H\&E staining ${ }^{26}$.

PA microscopy modalities offer distinct advantages as they capture optical absorption contrast. PA offers chromophore specific visualizations by leveraging the unique optical absorption spectra of biomolecules. This has been applied to selectively image DNA/RNA, hemoglobin, melanin, lipids, collagen, and more ${ }^{14,17,27-31}$. These unique chromophore specific visualizations position PA as a potentially powerful tool for label-free histological imaging ${ }^{14,15}$. The main challenge hampering the clinical adoption of this technique is that PA microscopy is a hybrid opto-acoustic modality. Conventional PA systems require physical contact with samples to perform imaging. However, a revolutionary new non-contact PA modality, Photoacoustic Remote Sensing (PARS) microscopy, has emerged as a frontrunner in label-free imaging $15,16,18,19,30,32$. Unlike traditional photoacoustic systems, PARS uses an all-optical pump-probe architecture. In PARS, the pump generates photoacoustic signals, which the probe captures as back-reflected intensity modulations ${ }^{30}$. Previously this technique was successfully applied to histology-like imaging in unprocessed resected tissue specimens, thin tissue sections, and paraffin embedded tissue blocks ${ }^{15,16,18,19,33}$. In these works, PARS provided histological visualizations analogous to hematoxylin staining of cell nuclei by leveraging the UV absorption contrast of DNA ${ }^{15,16,18,19,33}$. More recently, PARS hematoxylin-like imaging has been extended to full H\&E emulation ${ }^{15,19,34}$. These methods leveraged endogenous absorption ${ }^{15,19}$ and scattering contrast ${ }^{34}$ to capture extra-nuclear morphology to accompany the PARS nuclear visualizations. However, these methods cannot match the single acquisition volumetric imaging of extra-nuclear tissue structures provided by OCT.

This paper presents a conjoined PARS microscope and swept source OCT (SS-OCT) system for rapid 3D virtual histology in bulk unprocessed resected tissue specimens. The UV excitation PARS microscope is used to provide chromophore specific recovery of nuclear morphology. Concurrently, the SS-OCT is used to capture 3D volumetric images of tissue morphology. The co-registered PARS and OCT data are then merged to provide three-dimensional, histological, and structural tissue visualizations. While a combined PARS and OCT system has previously been proposed for ocular and vascular imaging ${ }^{35,36}$, this is the first such system for histopathology. Though, since the submission of this article, Martell et al. have presented a PARS-OCT histology system ${ }^{37}$. However, their technique does not provide co-registered PARS and OCT imaging or three-dimensional histology ${ }^{37}$. Applying the SS-OCT in resected Rattus tissues volumetric images containing adipocytes, ducts, fascia layers and other tissue features are recovered. Applying the PARS system in unprocessed resected human and Rattus tissues for the first time presents novel high spatial resolution imaging of predominately nuclear structures. Within the PARS visualizations tissue boundaries, and nuclear atypia, arrangement, organization, and density may be assessed. Moreover, the optical sectioning capabilities of the PARS microscope are leveraged to capture the first 3D volumetric images of subsurface nuclear morphology in unprocessed tissues. Together this system enables label-free non-contact assessment of nuclear structures directly on 3D visualizations of resected tissue specimens. These visualizations provide qualitatively similar diagnostic features to conventional $\mathrm{H} \& \mathrm{E}$ preparations. If employed in a clinical setting, the proposed technique could allow direct histology-like imaging of unprocessed resected tissue samples. Thus, the PARS-OCT system holds the potential to dramatically reduce current limitations in the histopathological processing of tissues. This could provide near-real time histological analysis, circumvent current diagnostic limitations, and greatly improve patient outcomes. 


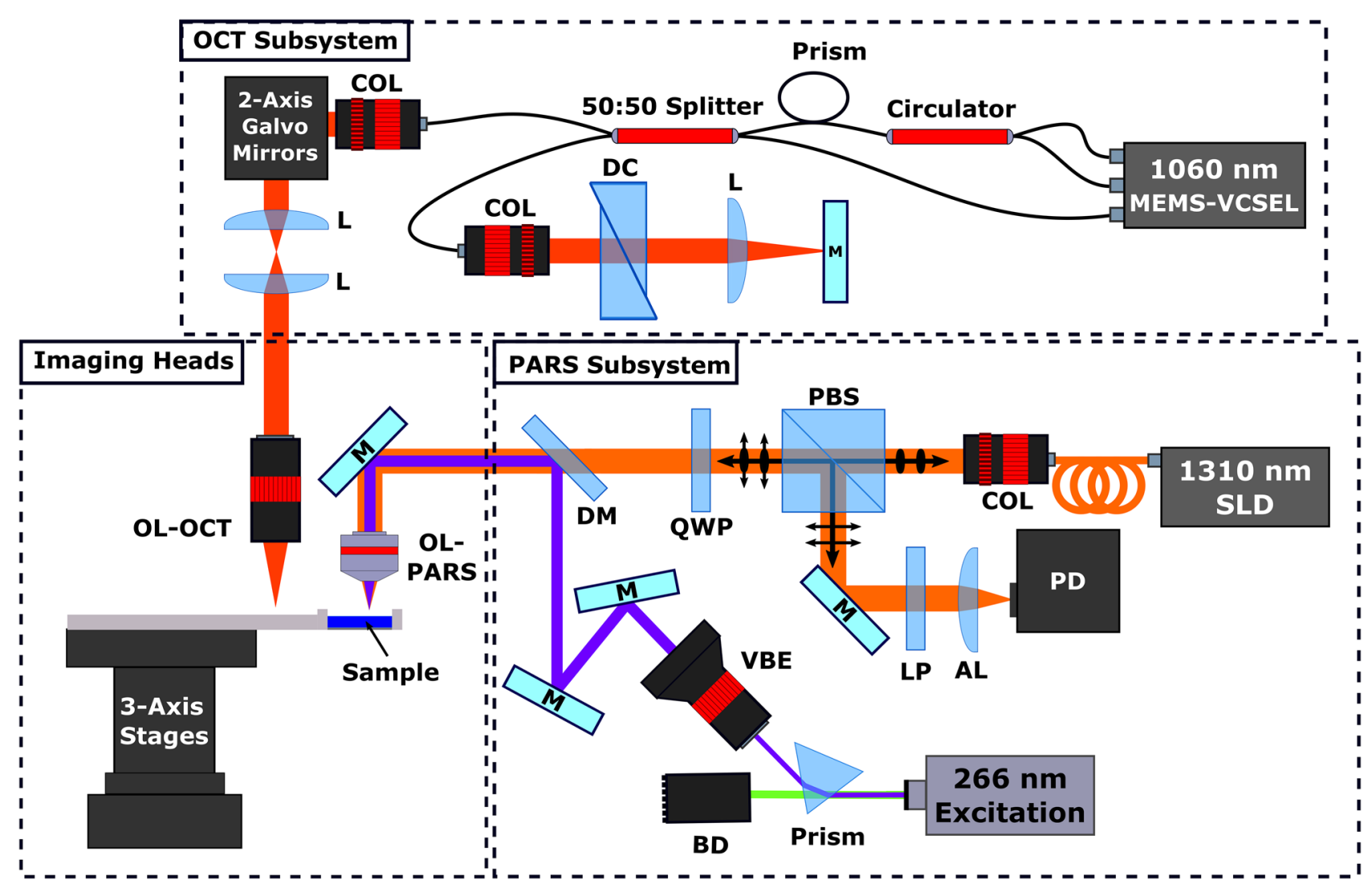

Figure 1. Simplified schematic of the combined OCT and PARS system. Component labels are defined as follows: OCT objective lens (OL-OCT), PARS objective lens (OL-PARS), collimator (COL), dispersion compensation (DC), lens (L), beam dump (BD), variable beam expander (VBE), polarizing beam splitter (PBS), quarter wave plate (QWP), dichroic mirror (DM), long pass filter (LP), aspheric focal lens (AL), photodiode (PD), $1310 \mathrm{~nm}$ super-luminescent diode (1310 nm SLD), mirrors (M).

\section{Methods}

Imaging system architecture. OCT system. The OCT system (Fig. 1) used a MEMS-VSCEL light source (Thorlabs, Inc.) centered at $\sim 1060 \mathrm{~nm}$ with $\sim 100 \mathrm{~nm}$ full width sweep bandwidth and $60 \mathrm{kHz}$ sweep rate. To synchronize data collection, A-line trigger, also known as wavelength sweep trigger, was supplied by the laser source. Concurrently, K-linear clock signals were provided by the Mach-Zehnder interferometer-based clock module integrated within the laser source. The swept source laser output was connected to a custom fiber optic interferometer. The interferometer consisted of a circulator and a 50:50 fiber splitter to direct light into the reference and sample arms. The reference arm consisted of a pair of BK7 dispersion compensating prisms, and a translating mirror to set the zero delay. In the sample arm, collimated light was directed through a pair of two-axis galvo-scanner mirrors, a 1:1 telecentric pair, then focused on the sample using an infinity corrected microscope objective. Light returning from the sample was coupled back into the 50:50 splitter where it was combined with the reference beam. The combined beams were detected by the swept sources built-in dual balanced photodetector. The OCT signals from the photodetector were then recorded with a high-speed 16-bit digitizer card (ATS9351, Alazar Technologies Inc., Pointe-Claire, QC, Canada).

Considering system performance, the maximum SNR of $105 \mathrm{~dB}$ was measured at $\sim 100 \mu \mathrm{m}$ away from the zero-delay line with incident power of $\sim 1.5 \mathrm{~mW}$. The SNR roll-off in free space was measured to be $\sim 1 \mathrm{~dB}$ over a scanning range of $1.3 \mathrm{~mm}$. Here, the $\mathrm{SNR}$ was calculated as a ratio of the maximum signal from a perfect reflector (mirror) versus the standard deviation of the background. The resolution of the OCT system was also determined as follows. The lateral resolution was characterized based on the 10 to 90 percent rise of the edge spread function (ESF) generated from imaging a USAF resolution target. The lateral resolution of the OCT is directly related to the numerical aperture of the objective lens. In this study, two lenses were used, a 0.4 NA and a 0.14 NA objective. Primarily the performance of the higher 0.4 NA lens was characterized (Fig. 2a). With this lens (0.4 NA), the lateral resolution was $\sim 3.9 \mu \mathrm{m}$ (Fig. $2 \mathrm{a}$ ). This was reduced to $\sim 15.6 \mu \mathrm{m}$ when using the lower 0.14 NA lens. Concurrently, the axial resolution was measured from the point spread function (PSF) generated from a perfect reflector (surface of the USAF resolution target) (Fig. 2b). The FWHM of the PSF was determined to be $10.1 \mu \mathrm{m}$ in free space (Fig. $2 \mathrm{~b}$ ), corresponding to $7.3 \mu \mathrm{m}$ in tissues, assuming an average refractive index of $\mathrm{n}=1.38$.

PARS system. For the PARS system (Fig. 1), UV excitation was provided by a $266 \mathrm{~nm} 400 \mathrm{ps} 50 \mathrm{kHz}$ pulsed laser (WEDGE XF, Bright Solutions). The $266 \mathrm{~nm}$ excitation was separated from residual $532 \mathrm{~nm}$ output using 
(a)

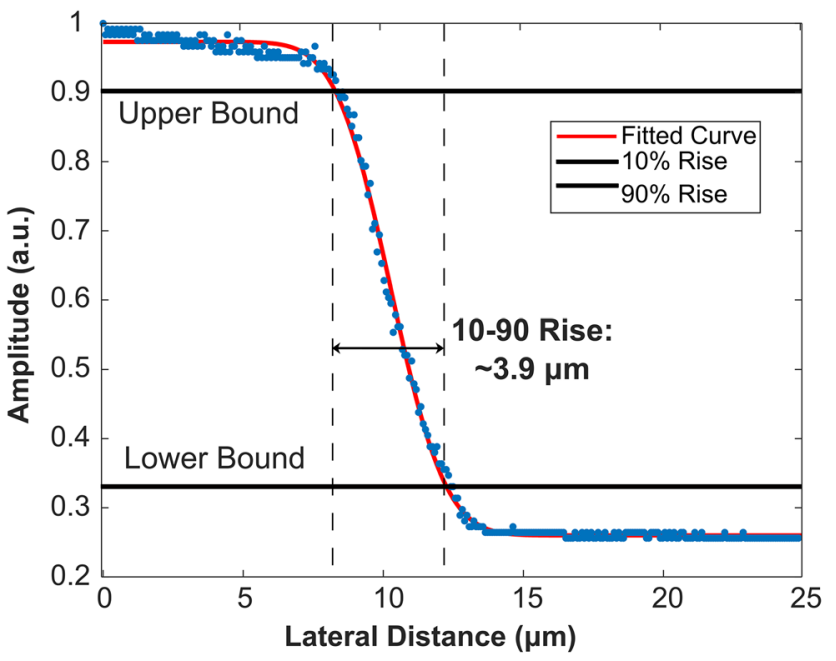

(b)

OCT Axial Point Spread Function

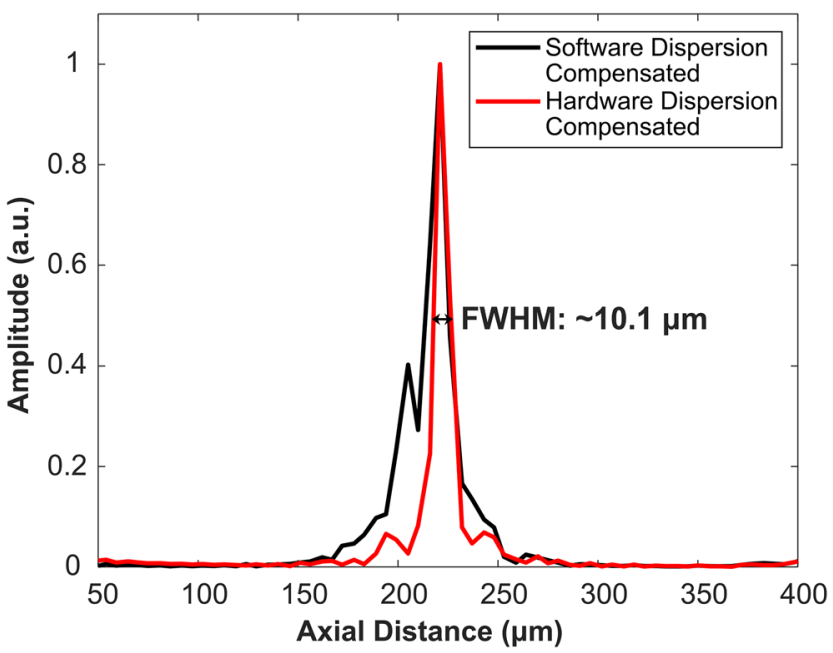

Figure 2. Imaging performance of the OCT subsystem. (a) Lateral edge spread function of the OCT scattering contrast mechanism characterized by imaging a USAF resolution target. OCT lateral resolution is measured to be $\sim 3.9 \mu \mathrm{m}$, with the $0.4 \mathrm{NA}$ objective lens. (b) Axial PSF of OCT subsystem measured in free space. OCT axial resolution is measured to be $\sim 10.1 \mu \mathrm{m}$.

(a)

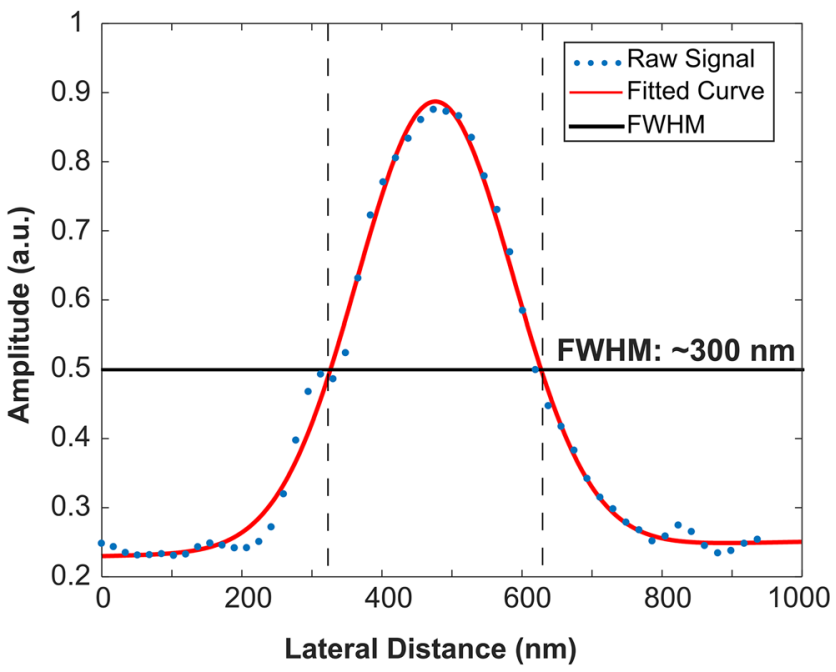

(b)

PARS Axial Edge Spread Function

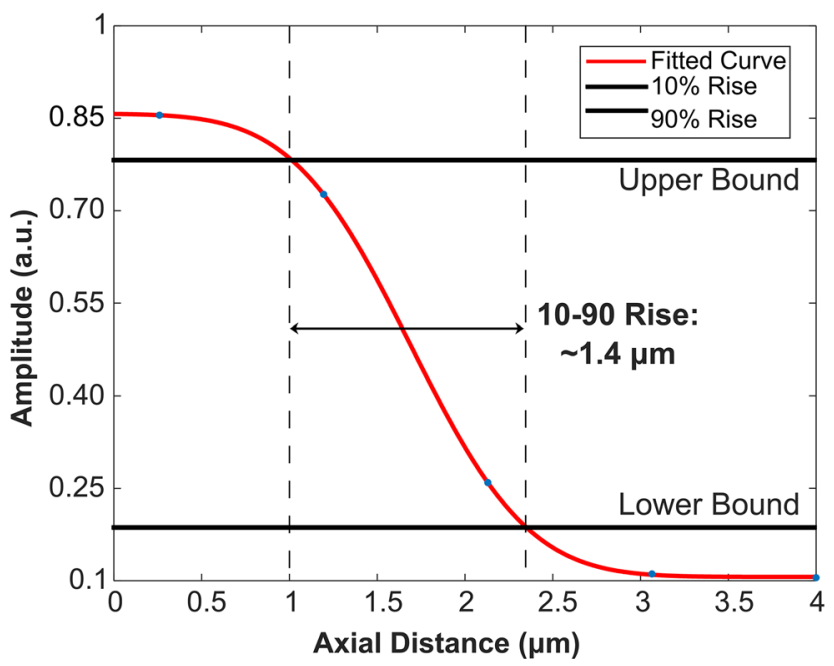

Figure 3. Imaging performance of the PARS subsystem. (a) Lateral PSF of the UV-PARS absorption contrast mechanism characterized by imaging $200 \mathrm{~nm}$ gold nanoparticles. PARS lateral resolution is measured to be $\sim 300 \mathrm{~nm}$. (b) Axial edge spread function of the UV-PARS absorption contrast mechanism modeled from volumetric imaging of cell nuclei. PARS axial resolution is determined to be $\sim 1.4 \mu \mathrm{m}$.

a CAF2 prism. Once isolated, the UV excitation beam was expanded and combined with the probe beam. The $1310 \mathrm{~nm}$ probe was supplied by a continuous wave super-luminescent diode laser from Thorlabs (S5FC1018P, Thorlabs). The horizontally polarized probe beam was passed through a polarizing beam splitter and quarter wave plate into the imaging system. The combined excitation and probe beam were then focused onto the sample using a $0.5 \mathrm{NA}$ reflective objective (LMM-15X-UVV, Thorlabs). The back-reflected probe beam containing the PARS signals returned to the quarter wave plate and polarizing beam splitter by the same path as forward propagation. Upon passing through the quarter wave plate a second time, the returning probe beam became horizontally polarized. The horizontally polarized probe beam was redirected down the detection pathway by the polarizing beam splitter. Here, the probe beam was isolated through spectral filtering, then focused onto a photodiode (PDB425C-AC, Thorlabs).

Considering system performance, the PARS resolution is defined by the focal spot size of the UV excitation beam. Therefore, the PARS may achieve sub-micron lateral and micron scale axial resolution absorption contrast using the 0.5 NA objective lens. The PARS lateral resolution was determined based on the PSF generated from 
imaging a $200 \mathrm{~nm}$ gold nanoparticle (Fig. 3a). Based on the FWHM, the lateral resolution is $300 \mathrm{~nm}$ (Fig. 3a). Concurrently, the PARS axial resolution was modeled from the PSF generated from $200 \mathrm{~nm}$ gold nanoparticles, and the ESF generated from volumetric imaging of cell nuclei (Fig. 3b). Based on the 10-to-90 percent rise of the ESF, the axial resolution was determined to be $\sim 1.4 \mu \mathrm{m}$ (Fig. $3 \mathrm{~b}$ ). These resolutions were determined in predominately surface and shallow subsurface structures. Therefore, it is expected the resolution will degrade below these benchmarks when imaging deep into tissues.

Imaging reconstruction and processing. OCT image reconstruction and processing. Raw OCT frames were captured by optically scanning the beam across the sample using the 2 -axis galvo-mirrors. At each wavelength sweep trigger, the interferogram and corresponding location were recorded. To capture the spectral interferogram 2448 sampling points were acquired with the 16-bit digitizer, providing a depth range of $\sim 12 \mathrm{~mm}$. Directly following acquisition, the OCT reference spectrum was subtracted from the interferogram to remove DC bias. The data was then Fourier transformed, to extract the depth-resolved OCT signal. The top half of the Fourier transformed data was considered as valid for further processing. To generate volumetric data sets, series of A-lines (usually 500 to 800) were acquired to form a cross sectional B-scan. Series of adjacent B-scans (usually 500-800) were collected to form a volumetric data set. Once the entire volume was captured, the raw OCT data was numerically dispersion compensated up to the 5th order with a custom MATLAB algorithm. No additional post-processing was applied to the OCT results presented in this paper. Volumetric and enface images were then generated from the $3 \mathrm{D}$ data sets with Image ${ }^{38}$.

PARS image reconstruction and processing. To form PARS images the co-focused excitation and probe beams were mechanically scanned across the sample. During scanning, the excitation laser was pulsed continuously, interrogating regularly spaced points of absorption contrast. The lateral spacing of the interrogation points was modified by adjusting the stages mechanical scanning pattern and speed. In this study, most images were collected with $500 \mathrm{~nm}$ lateral spacing. At each interrogation event, the PARS signal, and interrogation location are recorded. These signals were captured using a high bandwidth 14-bit digitizer (RZE-004-300, Gage Applied). Location signals were recorded directly from the mechanical stages, while PARS signals were recorded by capturing a $\sim 500$ ns segment of photodiode signal. The digitized signals were then streamed directly to the computer memory. Processing was applied to compress the time domain data, to a single characteristic PARS amplitude and corresponding location signal. Once scanning was complete, the scattered data was reconstructed into an image. Here, the location signals were used to impose the PARS signals onto a cartesian grid, forming a raw PARS image. Raw data was gaussian filtered to reduce scanning noise, then normalized and log scaled to convert the PARS signals to a decibel scale. To generate the final image, the logarithmic PARS image was rescaled based on histogram distribution to reduce background noise.

PARS and OCT image co-registration. Combined PARS and OCT visualizations were generated using the final PARS images, and processed OCT volumetric data. Prior to processing, the PARS images were overlayed over the max amplitude projection of the OCT volume to manually tune the co-registration, as there were some minor motion artifacts. Since the PARS images were substantially higher resolution than the OCT frames, the datasets had to be rescaled prior to combination. For the large area scans, the PARS image was compressed to match the OCT volume. Conversely, for the high-resolution frames, the OCT volume was interpolated to match the PARS image dimensions. Merging the PARS data into the OCT volume was performed one B-scan at a time. In each B-scan, the tissue surface was extracted based on the regional maximum of the OCT signal. The corresponding stripe of PARS contrast was then merged into the OCT B-scan along the extracted surface. The entire volumetric dataset was processed in this fashion. Volumetric and enface images were generated from the resulting $3 \mathrm{D}$ data sets with Image ${ }^{38}$.

Sample preparation. Tissue samples were collected under protocols approved by the Research Ethics Board of Alberta (Protocol ID: HREBA.CC-18-0277) and the University of Waterloo Health Research Ethics Committee (Humans: \#40275, and Animals: \#41543). During this study, informed consent was waived by the Research Ethics Board of Alberta and the University of Waterloo Health Research Ethics Committee as samples were archival tissue not required for diagnostic purposes, and no patient identifiers were provided to the researchers.

All experiments involving human tissues were carried out in accordance with the government of Canada guidelines and regulations, such as "Ethical Conduct for Research Involving Humans (TCPS 2)". All experiments involving animal tissues were carried out in accordance with the government of Canada guidelines and regulations on research involving animals. Where applicable, ARRIVE reporting guidelines were observed.

Thin tissue sections. Thin sections of human breast tissue were used to provide a one-to-one comparison between PARS imaging and H\&E staining. Tissue specimens were collected as follows. Breast tissue samples were excised from a patient and placed into a $10 \%$ formalin solution within 20 min of extraction. Samples were soaked for $24 \mathrm{~h}$ to ensure full formalin fixation. Once fixed, samples were embedded into a paraffin wax substrate. These embedded sections are known as Formalin Fixed Paraffin Embedded (FFPE) human tissue blocks. Several $4 \mu \mathrm{m}$ sections were cut from the FFPE tissue blocks using a microtome. Each thin section was fixed to a slide. Unstained tissue slides were transported to the PhotoMedicine Labs and imaged with the PARS microscope. Following PARS imaging, the unstained slides were returned to the clinicians to undergo traditional histopathological staining. Here, the slides were stained with hematoxylin and eosin dyes, then completed with mounting media and a cover slip. Once completed, slides were imaged with a digital pathology scanner. With 
this method, the exact same section of tissue was captured with PARS microscopy and traditional H\&E staining, thus providing a direct one-to-one comparison between PARS and the current gold standard for histopathological imaging.

Bulk tissues. Both human and Rattus mammary tissues were explored during this study. Human tissue samples were collected from a post-menopausal breast during a tumor excision surgery. Rattus tissue samples were collected from rats with naturally occurring mammary tumors. Rats found to have tumors were sacrificed according to the endpoint protocols laid out by the University of Waterloo Research Ethics committee (Animals: \#41543). Following sacrifice, the mammary tumors were extracted.

In both cases, mammary tissues were resected and placed directly into a fixative solution of $10 \%$ formalin within 20 min of resection. Fixed tissues were transported directly to the PhotoMedicine labs at the University of Waterloo. Prior to imaging, the bulk tumors were sectioned to expose regions of dense tumor tissue. Then, the unstained bulk tissues were placed directly onto the PARS and OCT system, and imaged at room temperature. Following imaging, the samples were returned to their formalin storage containers.

\section{Results and discussion}

To capture nuclear contrast, the PARS system targets the optical absorption peak of DNA using a $266 \mathrm{~nm}$ UV excitation. Prior to testing the proposed system within resected human tissues, the UV-PARS contrast was validated in thin tissue sections. Here, an entire unstained FFPE thin human breast tissue section was scanned with the PARS system. Once imaged with the PARS microscope, the same tissues were stained with H\&E dyes. The freshly stained tissues were then scanned with a digital pathology system. The resulting PARS and H\&E images are presented in Fig. 4i, ii respectively. Observing the wide field PARS image (Fig. 4a-i), this scan captures nearly an entire resected breast tissue slide. The $9 \mathrm{~mm} \times 13.25 \mathrm{~mm}$ PARS scan was captured in high resolution at a rate of $\sim 90 \mathrm{~s}$ per $\mathrm{mm}^{2}$ (Fig. $4 \mathrm{a}-\mathrm{i}$ ), resulting in a 780-megapixel final image. Here, the PARS UV contrast provides visualizations analogous to hematoxylin staining of cell nuclei. Observing the large field PARS scan and corresponding H\&E image, broad features such as nuclear density, arrangement, size, and organization may be assessed. Based on these characteristics, analogous regions of tissues can be identified in each the PARS and $\mathrm{H} \& \mathrm{E}$ image. To further assess the PARS and H\&E concordance, several smaller subsections of the large field image are evaluated in Fig. 4a. Within these subframesnuclei to nuclei agreement between the two modalities is observed. Identical nuclear structures are highlighted by both the PARS and H\&E process. Moreover, each the PARS and H\&E images provide nearly identical diagnostic criteria enabling assessment of the tissues in each subframe. Both modalities reveal invasive ductal carcinoma with background inflammation, as seen by the abundance of lymphocytes (Fig. 4b). Dedifferentiated sheets of ductal carcinoma indicating high grade disease with minimal cribriform pattern are also seen (Fig. 4c). Invasive ductal carcinoma with aberrant pleiomorphic nuclei organizing into glandular structures, more indicative of a region of intermediate grade disease are detected (Fig. 4d). Accompanying this intermediate disease region, reactive stroma around the neoplastic regions with lymphocyte infiltration are also observed (Fig. 4d). Each the PARS and H\&E images provide essentially identical nuclear visualizations, thus providing access to the criteria required to make diagnostic decisions. Moreover, it can be concluded that the UV-PARS may be applied to unstained tissues to provide visualizations analogous to those of hematoxylin staining of cell nuclei.

Applying PARS to unprocessed resected tissues captures high-fidelity visualizations of nuclear structures, analogous to hematoxylin staining of cell nuclei. Such visualizations are shown in Fig. 5. Here, bulk sections of formalin fixed tissues were placed directly onto the PARS imaging stage, then scanned with the PARS system. These images reveal nuclear structures directly within unprocessed formalin fixed Rattus tissues, and for the first time, unprocessed human breast tissues. Within the presented images, one can discern and differentiate clinically relevant diagnostic features of the nuclear morphology. For example, in Fig. 5a-i, highly cellular regions and the surrounding nuclear sparse adipose and connective tissues can be differentiated. Observing a subsection of this large field image, Fig. 5a-ii enables discernment of subtler diagnostic details. Within the higher magnification region, cellular structures organized into a circular morphology indicating mammary glands and ducts can be identified. Looking at a smaller subsection of the large field, Fig. 5a-iii, highlights some of the gland and duct architecture and the boundary between the nuclear dense tissues (bottom right) and the sparse surrounding regions (top left). This small frame exemplifies the $\sim 300 \mathrm{~nm}$ lateral resolution (measured on $200 \mathrm{~nm}$ gold nanoparticles) provided by the PARS system. Within the small frame visualization of individual cells' nuclei and how they are arranged in the tissue architecture is accessible. Applying this technique to human tissues for the first time, achieves similar diagnostic quality (Fig. 5b-d). Within the human tissues, Fig. $5 \mathrm{~b}$ multiple regions of dense nuclear structures, and the surrounding connective tissues are discerned. Looking closer at the hypercellular regions (Fig. 5c, d), individual cells and nuclei become appreciable as well as their distribution within the tissue sample.

In addition to high lateral resolution, the proposed PARS microscope provides high axial resolution. In this architecture, the PARS system provides a $\sim 1.4 \mu \mathrm{m}$ focal plane. Leveraging this tight axial focus, the PARS system may perform optical sectioning to capture isolated planes of depth resolved nuclear contrast within excised specimens (Fig. 6a). Here, the PARS captured a series of nuclear visualizations from the sample surface through to $\sim 30 \mu \mathrm{m}$ in depth. This image series was then reconstructed to form a $3 \mathrm{D}$ representation of the subsurface nuclear morphology (Fig. 6b-d). In this volumetric reconstruction, the PARS resolves dense layers of nuclei representing the edge of the tissues. Adjacent to this, multiple overlapping layers of more sparse nuclear structures within the tissue specimens may be seen. Successively deeper layers in the $3 \mathrm{D}$ reconstruction also reveal subsurface cellular morphology of ductal and glandular breast tissue. These results represent the first report of a non-contact photoacoustic system capturing $3 \mathrm{D}$ volumetric images of subsurface nuclear morphology in resected 

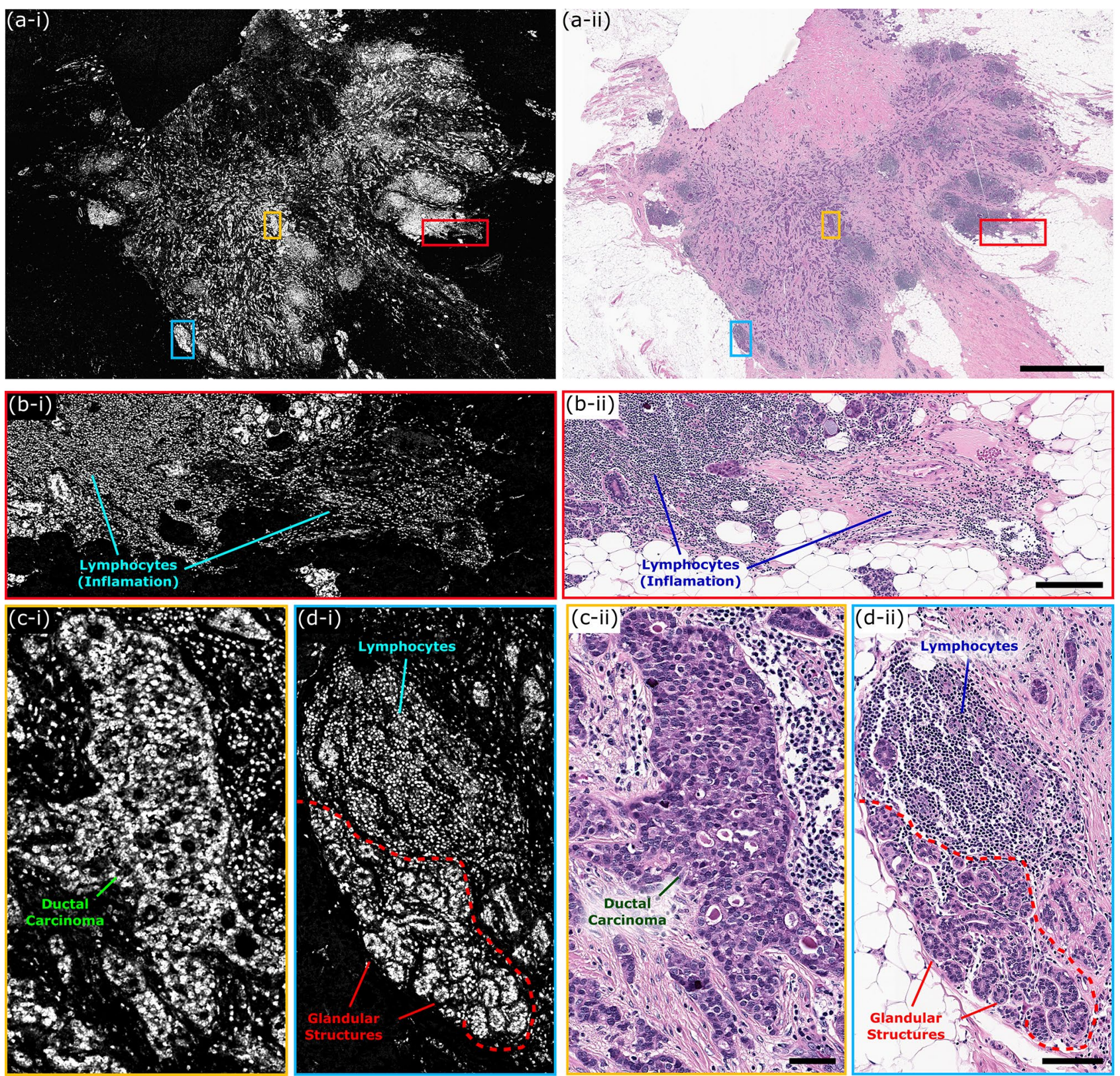

Figure 4. One to one comparison of PARS histological imaging and H\&E staining. (a) Large field of view $(9 \mathrm{~mm} \times 13.25 \mathrm{~mm})$ PARS (780 megapixel) image of nuclear morphology in a thin section of formalin fixed paraffin embedded excised breast tumor tissues. (b-d) (i) Subsections of the large field of view PARS image (a) highlighting regions of interest within the large field scan. (ii) Images of the exact same set of tissues following $\mathrm{H} \& \mathrm{E}$ staining, providing a direct one-to-one comparison between PARS imaging of tissues and the gold standard for histopathology H\&E imaging. (a) Scale Bar: 2 mm. (b) Scale Bar: $200 \mu \mathrm{m}$. (c) Scale Bar: $50 \mu \mathrm{m}$. (d) Scale Bar: $75 \mu \mathrm{m}$.

tissues. Compared to the $5 \mu \mathrm{m}$ thickness of current paraffin tissue preparations, PA based histopathology systems have been shown to image $\sim 100 \mu \mathrm{m}$ into resected tissue specimens ${ }^{14,17}$. As the UV excitation is highly absorbed and scattered within tissues, PA systems begin to rapidly lose contrast and resolution beyond this depth. While the imaging depth could be increased by using larger UV excitation pulses, this may result in unsafe UV exposure to the sample. Generally, to image tissue surfaces (as in Figs. 4 and 5), $0.8 \mathrm{~nJ}$ UV excitation pulses were used. In this case, when performing subsurface imaging the excitation pulse energies were increased to a maximum of $25 \mathrm{~nJ}$ resulting in a penetration depth of $\sim 30 \mu \mathrm{m}$. However, even with this limitation the PARS virtual sectioning captures nuclear morphology equivalent to the depth provided by $\sim 6$ thin tissue preparations. Applied in a clinical setting, the PARS virtual sectioning capabilities would allow pathologists to rapidly assess subsurface nuclear structures without extensive sample processing.

To compliment the nuclear morphology captured by the UV excitation PARS system, OCT is applied to image the bulk tissue structures, and subsurface specimen morphology. The proposed OCT subsystem provides 

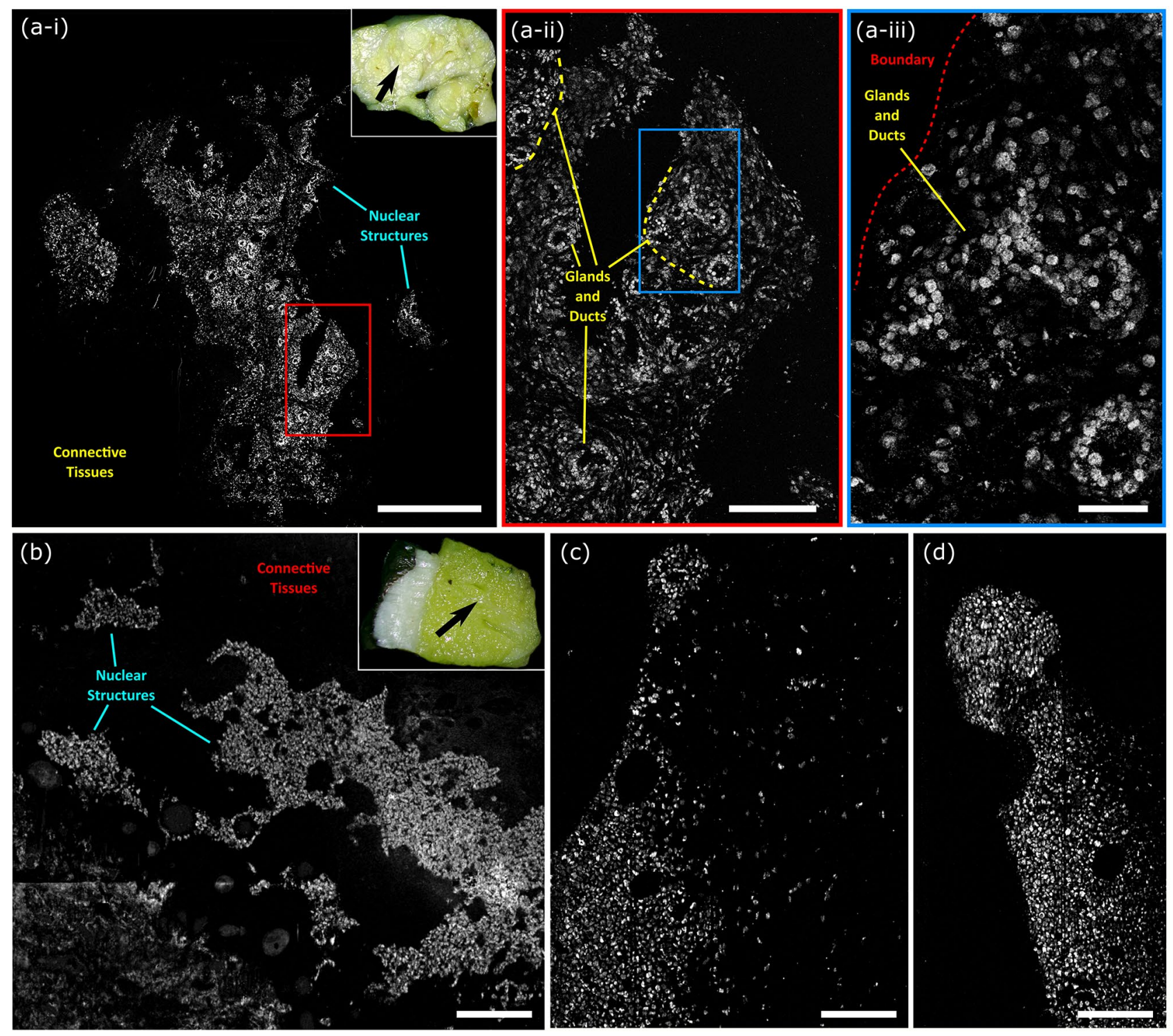

Figure 5. PARS histological imaging of nuclear morphology in unprocessed excised tumor tissues. (a) (i) Large field of view PARS image of resected Rattus mammary tumor. The inset in the upper right indicates the region of the bulk tissue section in which the PARS images were captured. Scale Bar: $1 \mathrm{~mm}$. (ii, iii) Subsections of the large field of view image in (i) highlighting regions of interest within the large field images. (ii) Scale Bar: $500 \mu \mathrm{m}$. (iii) Scale Bar: $25 \mu \mathrm{m}$. (b-d) PARS histological imaging of nuclear morphology in unprocessed formalin fixed excised post-menopausal human breast tissues. The inset in the upper right highlights the region of the breast tissue section which the PARS images were captured in. (b) Scale Bar: $275 \mu \mathrm{m}$ (c) Scale Bar: $150 \mu \mathrm{m}$ (d) Scale Bar: $150 \mu \mathrm{m}$.

$10.1 \mu \mathrm{m}$ axial resolution in free space, corresponding to $7.3 \mu \mathrm{m}$ in tissues, assuming an average refractive index of $\mathrm{n}=1.38$. Depending on the application, the system used either a 0.14 NA or 0.4 NA objective corresponding to $\sim 15.6 \mu \mathrm{m}$ and $\sim 3.9 \mu \mathrm{m}$ lateral resolution, respectively. The lower $0.14 \mathrm{NA}$ lens provided wide area grossing scans capturing nearly $1 \mathrm{~cm}^{2}$, while the higher 0.4 NA lens provided high resolution imaging of $\sim 0.5 \mathrm{~mm}^{2}$ fields. In resected tissue specimens, the OCT system provided visualizations as presented in Fig. 7. Large field scans capture entire tumor specimen, providing a 3D representation of the bulk tissue structure (Fig. 7a). Leveraging the unique depth resolved scattering contrast provided by OCT, enables assessment of the bulk specimens subsurface tissue morphology, Fig. 7b-i, b-ii. Observing the subsurface structures, regions of breast connective and adipose tissue, fibrous structural tissue, and gland/duct tissue become evident. Changing over to the 0.4 NA lens, the OCT captured smaller high-resolution visualizations of tissue structures. Several max projection images of small fields are shown in Fig. 7c-e. Within these images, subtler features such as connective tissue bands segmenting structures within the tissue (Fig. 7c), with potential spaces of ducts and glands interspersed are discerned. In other regions, circular voids within tissues adjacent to bands of connective tissues are identified (Fig. 7d). Moreover, less feature rich regions are captured likely representing adipose tissue (Fig. 7e). Both the large and small field visualizations highlighted here, provide analogous contrast and quality to other implementations of 

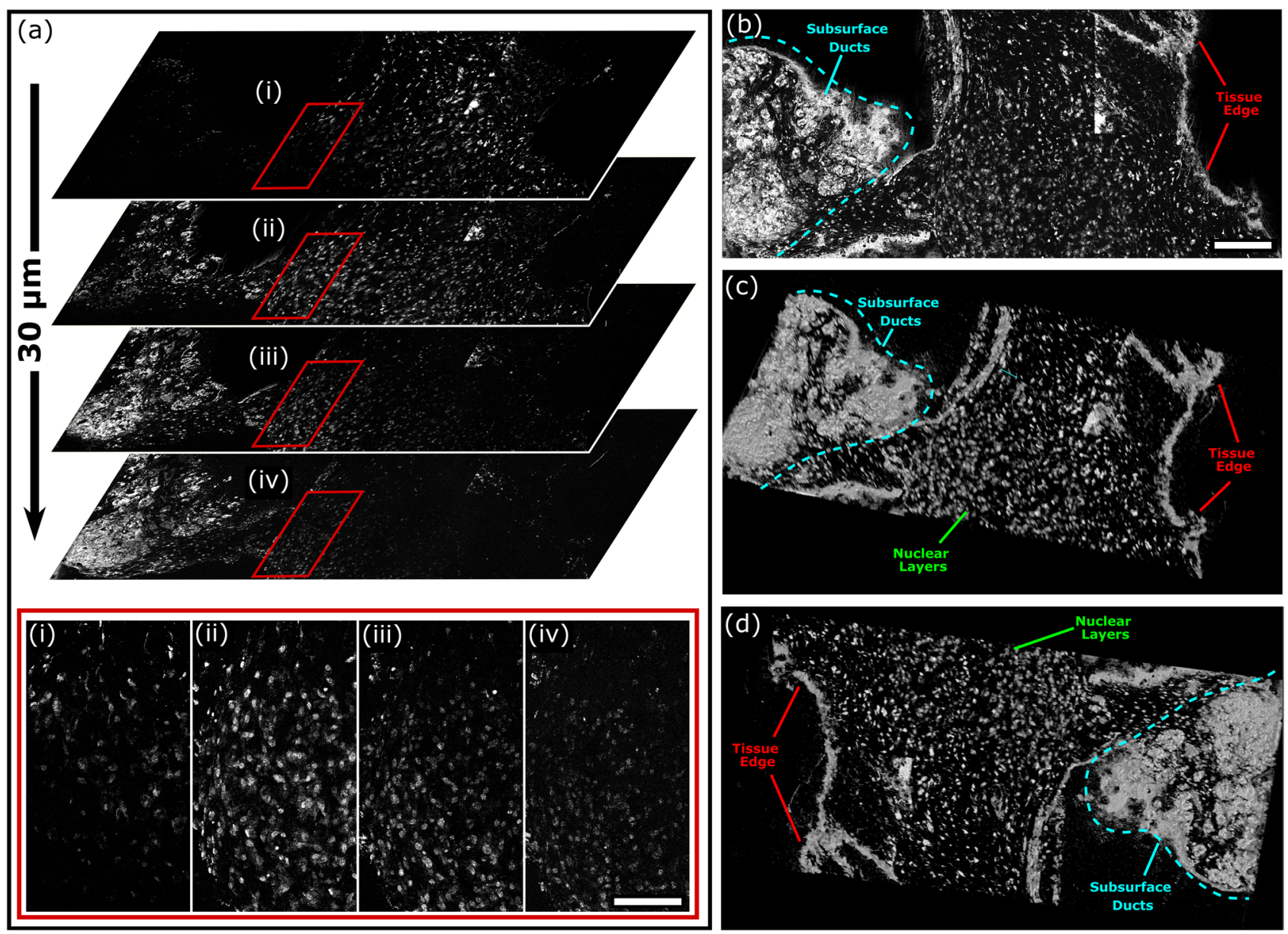

Figure 6. PARS 3D volumetric imaging of subcellular nuclear morphology in unprocessed excised Rattus mammary tumor tissue. (a) A subset of the optically sectioned volumetric image stack showing subsurface nuclear morphology from the surface to $30 \mu \mathrm{m}$ into the tissue. Scale Bar: $125 \mu \mathrm{m}$. (b-d) Different angular views of the 3D reconstruction of the nuclei within the excised tissue sample. Scale Bar: $200 \mu \mathrm{m}$.

OCT imaging in resected tissues ${ }^{37,39-41}$. As with previous reports ${ }^{37,39-41}$, OCT may highlight different regions of tissue, and tissue structures, however, cannot discern nuclear structures.

In isolation, PARS and OCT each present distinct advantages and weaknesses. The OCT provides unique depth resolved tissue morphology imaging; however, it cannot accurately highlight nuclear structures. Conversely, PARS provides chromophore specific visualizations readily capturing nuclear contrast, however, it cannot provide depth resolved tissue morphology in a single acquisition. Though, Bell et al. have proposed a coherencegated PARS microscope which could provide single acquisition depth resolved chromophore specific tissue imaging in the future ${ }^{42}$. In the current implementation, the PARS and OCT are combined to leverage the advantages of each system. This dual modality PARS and OCT system provides a unique suite of clinically relevant imaging capabilities. To capture combined PARS and OCT images, tissues were first scanned with the PARS microscope Fig. $8 \mathrm{a}$-i. The wide field PARS image captured here covers $\sim 50 \%$ of the resected tissue specimen and highlights the nuclear structures of the tissue. Once PARS imaging was complete, tissues were imaged with the OCT system equipped with the $0.14 \mathrm{NA}$ objective. The corresponding 3D OCT representation of the bulk tissue surface morphology is highlighted in Fig. 8a-ii. The co-registered datasets were merged to provide PARS images of nuclear morphology directly on the 3D OCT tissue visualizations, Fig. 8a-iii. These combined visualizations reveal the correlation between the PARS nuclear features, and the corresponding OCT based tissue morphology.

In practice, the PARS nuclear contrast was merged directly into the OCT volumetric data. Therefore, the combined PARS and OCT volumetric scans could be treated analogous to pure OCT images. This means 3D representations of tissues exhibiting nuclear morphology can be assessed from any angle and orientation, Fig. 8b, c. Moreover, since the PARS data was added to the OCT volume, the subsurface tissue morphology could still be assessed as with traditional OCT volumes, Fig. 8d. Limited rendering resolution was the main challenge associated with these 3D PARS and OCT visualizations. During data collection, the OCT scans were restricted to a resolution of $\sim 800$ by 800 pixels, due to the large volume of data. Therefore, in order to merge the PARS and OCT visualizations, the PARS data was down sampled to match the spatial resolution of the OCT. As a result, only the general cellular organization based on the nuclear contrast data could be resolved within the wide field representations. In order to view the nuclear morphology with higher resolution, subsections of interest from the large frame were re-imaged with the higher resolution 0.4 NA OCT. This allowed high resolution visualizations 

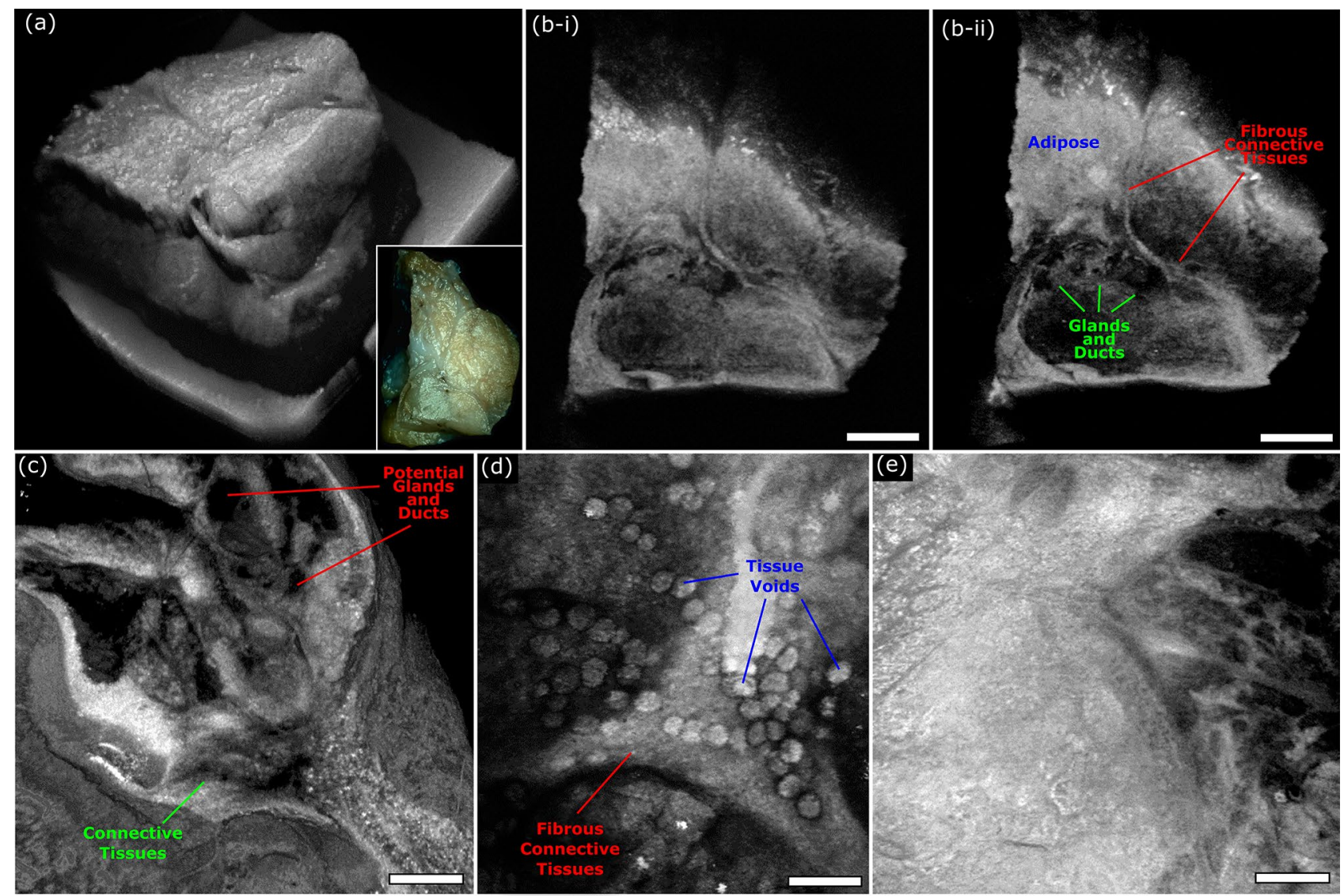

Figure 7. OCT imaging of unprocessed excised Rattus mammary tumor tissue. (a) Shows a large field OCT image capturing the surface morphology of an entire excised tumor specimen. The inset in the lower right shows a camera image of the same section of tissues. (b) Vertical sections showing subsurface tissue morphology of the tissue captured in (a). Scale Bar: $1.25 \mathrm{~mm}$. (c, d) Small field maximum projection images of different regions of excised Rattus mammary tissues captured using the OCT system. (c) Scale Bar: $100 \mu \mathrm{m}$. (d) Scale Bar: $100 \mu \mathrm{m}$. (e) Scale Bar: $100 \mu \mathrm{m}$.

of nuclear morphology directly on the OCT visualization Fig. 8e. Within these subframes, (Fig. 8e-h), areas of subcutaneous tissue demarked by more homogenous and smaller nuclei transitioning into more characteristic breast tissue architecture with duct and gland formation consisting of larger cells and nuclei are seen (Fig. 8e). Intervening adipose and connective tissue space is seen in Fig. $8 \mathrm{f}-\mathrm{g}$ between glandular breast tissue regions, with medium and small-sized blood vessels becoming evident (Fig. 8g). Observing even smaller subsections Fig. 8h, reveal sparsely distributed nuclei within the connective and adipose tissue where individual nuclear atypia can be assessed. This type of diagnostic interpretation can be ascertained more reliably with the context of both the high-resolution combined PARS and OCT renderings providing information (Fig. 8e-h). Applied in a clinical setting this would provide clinicians with visualizations of useful histopathology directly on excised tissues. This technique may potentially provide a more accurate representation of the true tissue margin compared to $\mathrm{H} \& \mathrm{E}$ preparation, since there is no requirement to section the tissue samples. Moreover, this should expedite histopathological assessment by circumventing current sectioning and staining requirements.

Moving forwards, several technical challenges must be overcome to further improve the clinical functionality of the proposed system. One of the main limitations of the current report was the $50 \mathrm{kHz}$ PARS excitation. With the existing UV source,imaging speed was limited to $\sim 90 \mathrm{~s}$ per $\mathrm{mm}^{2}$. To reduce imaging times, a new $2.7 \mathrm{MHz}$ excitation source is being implemented. The new source will reduce imaging times to a few seconds per square millimeter. This will enable real-time PARS histological imaging, and near real-time volumetric imaging. Another challenge which arises with the current implementation is the specificity of the combined contrast. While the PARS and OCT system recover nuclear morphology and tissue structures, the scattering contrast of OCT does not provide tissue specificity. Therefore, it may be difficult to differentiate extranuclear biomolecules such as collagen or lipids. To resolve this issue, it may be advantageous to add further PARS excitation wavelengths to the proposed system. More excitation wavelengths would expand the chromophore specific absorption contrast. This may provide visualizations of biomolecules such as lipids, collagen, heme proteins and more ${ }^{15,19,28,43}$.

Several refinements and adaptations could also be made to the OCT subsystem. The swept source OCT only provided $\sim 7.3 \mu \mathrm{m}$ axial and $\sim 3.9 \mu \mathrm{m}$ lateral resolution. This is substantially less than the $\sim 1.4 \mu \mathrm{m}$ axial and $\sim 300 \mathrm{~nm}$ lateral resolution of the PARS system. As a result, the OCT subsystem could not resolve structures on the same scale as the PARS microscope. This challenge is compounded when considering the PARS system 

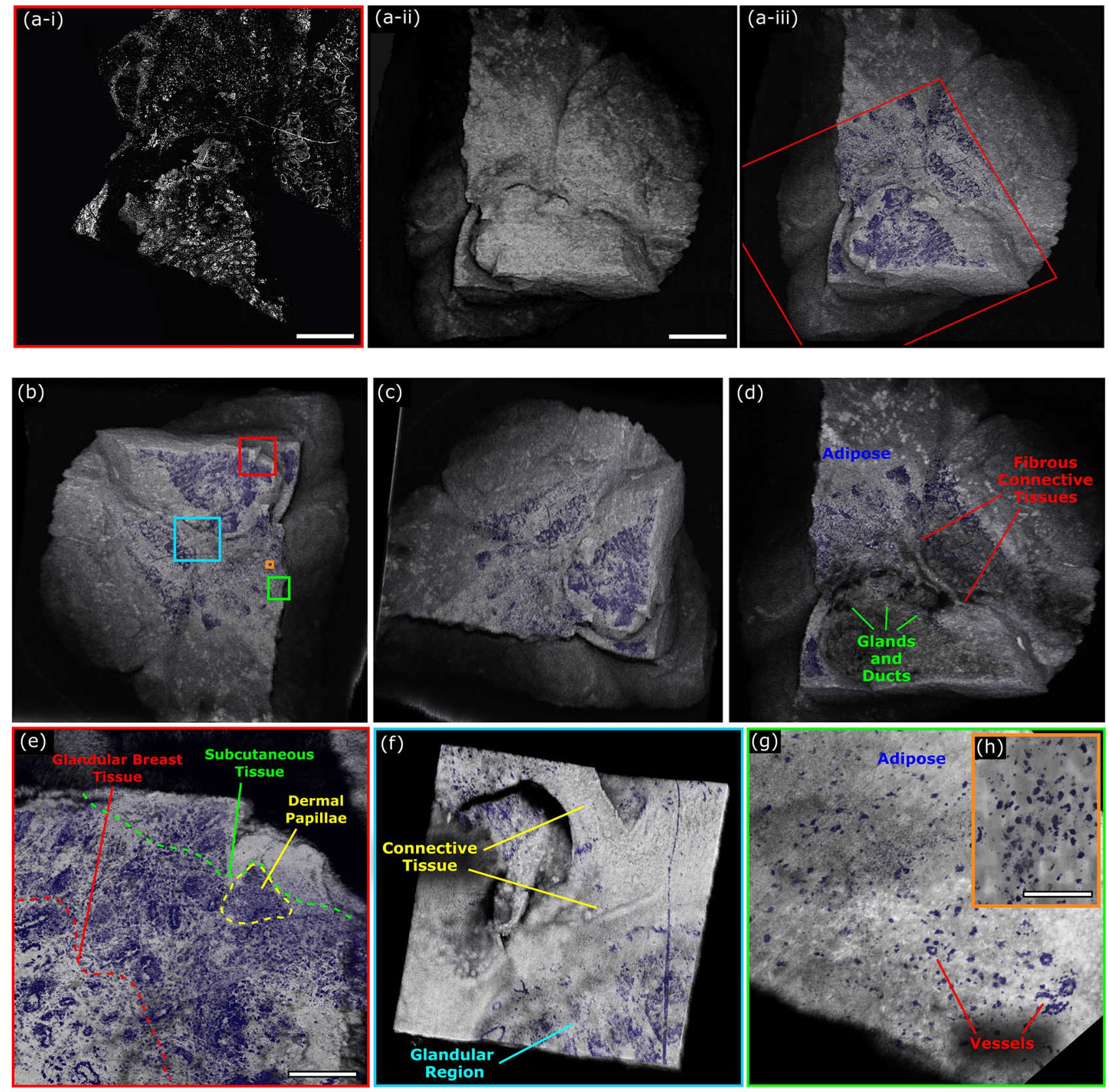

Figure 8. Combined PARS and OCT imaging of unprocessed excised Rattus mammary tumor tissue. (a) (i) PARS image of resected Rattus mammary tumor. Scale Bar: $1 \mathrm{~mm}$. (ii) OCT (0.14 NA) image of entire section of resected Rattus mammary tumor tissues. Scale Bar: $1.25 \mathrm{~mm}$. (iii) Merged PARS and OCT image of resected Rattus mammary tumor. Red box outlines limits of PARS image. (b, c) Different angles of 3D representation of merged PARS and OCT images of excised Rattus mammary tumor tissue. (d) Vertical subsection of PARS and OCT image showing subsurface tumor tissue morphology and nuclear visualizations. (e-h) Merged PARS and OCT (0.4 NA) image of resected Rattus mammary tumor. (e) Scale Bar: $250 \mu \mathrm{m}$. (h). Scale Bar: $100 \mu \mathrm{m}$.

may only provide volumetric imaging to $\sim 100 \mu \mathrm{m}$ deep ${ }^{14,17}$. Moving forwards, it may be advantageous to develop a higher axial resolution OCT system. If the PARS and OCT axial resolution were equal, the combined threedimensional representations could capture co-registered depth resolved images of subcellular structures including single nuclei. Another challenge to consider is the PARS and OCT subsystems use independent objective lenses. As a result, the co-alignment between PARS and OCT images was not perfect as tissues can potentially shift when switching between objectives. In the ideal case, rather than imaging with PARS and OCT independently, both images would be captured simultaneously through the same lens. This could significantly reduce imaging time. Moreover, if the PARS and OCT images were collected together, the OCT A-lines could be used to determine the height of the tissue surface at each location. An adaptive optics, or mechanical scanning system could then adjust the PARS system focus based on the OCT A-line to guide imaging of rough tissue surfaces. 


\section{Conclusions}

This work represents the first report of: (1) label-free histological imaging in human tissue specimens with a non-contact photoacoustic system, (2) 3D imaging of subsurface nuclear morphology in resected tissue specimen with a non-contact photoacoustic system, and (3) a combined photoacoustic and OCT microscope for label-free visualizations of nuclear and extranuclear morphology in resected tissue specimens. The proposed multimodal microscope provides co-registered sub-micron depth-resolved nuclear imaging, and micron scale depth resolved tissue morphology in an all-optical reflection mode format. These visualizations may be recovered label-free directly from thick unprocessed tissues. The clinically relevant diagnostic features captured by the proposed system are not accessible through any other means. The unparalleled clinical diagnostic potential of this system could revolutionize the workflow for diagnosis, treatment, and resection of cancer and other neoplasms. Applied in a clinical setting, the proposed system would allow pathologists to rapidly assess subsurface nuclear structures without extensive sample processing and reduce the potential for sampling error in larger pathology specimens. This technique may potentially improve diagnostic assessment of tissue margins compared to traditional H\&E preparation, since the tissue margin can be imaged without sectioning or processing. Bulk tissues could undergo histological imaging immediately after excision. This would improve the diagnostic yield of histological preparations, while reducing histological imaging timelines from weeks to minutes. Moreover, this would preserve whole tissues for further histopathological processing and immunochemistry. Moving forwards, this technology will soon be applied to imaging a wider variety of resected human tissues.

\section{Data availability}

All data generated or analyzed during this study are included in this published article.

Received: 23 April 2021; Accepted: 16 June 2021

Published online: 02 July 2021

\section{References}

1. Canene-Adams, K. Chapter fifteen. Preparation of formalin-fixed paraffin-embedded tissue for immunohistochemistry. Methods Enzymol. 533, 225-233 (2013).

2. Fischer, A. H., Jacobson, K. A., Rose, J. \& Zeller, R. Hematoxylin and eosin staining of tissue and cell sections. Cold Spring Harb. Protoc. 2008, pdb.prot4986 (2008).

3. Valejo, F. A. M., Tiezzi, D. G., Mandarano, L. R. M., de Sousa, C. B. \& de Andrade, J. M. Volume of breast tissue excised during breast-conserving surgery in patients undergoing preoperative systemic therapy. Rev. Bras. Ginecol. E Obstet. 35, 221-225 (2013).

4. Altaleb, A. A primer on gross pathology examination and selected images of gross specimens. In Surgical Pathology: A Practical Guide for Non-Pathologist (ed. Altaleb, A.) 49-66 (Springer, 2021). https://doi.org/10.1007/978-3-030-53690-9_9.

5. Fereidouni, F. et al. Microscopy with ultraviolet surface excitation for rapid slide-free histology. Nat. Biomed. Eng. 1, 957-966 (2017).

6. Crosignani, V. et al. Deep tissue fluorescence imaging and in vivo biological applications. J. Biomed. Opt. 17, 116023 (2012)

7. Yick, C. Y., von der Thüsen, J. H., Bel, E. H., Sterk, P. J. \& Kunst, P. W. In vivo imaging of the airway wall in asthma: Fibered confocal fluorescence microscopy in relation to histology and lung function. Respir. Res. 12, 85 (2011).

8. Olson, E., Levene, M. J. \& Torres, R. Multiphoton microscopy with clearing for three dimensional histology of kidney biopsies. Biomed. Opt. Express 7, 3089-3096 (2016).

9. Rogart, J. N. et al. Multiphoton imaging can be used for microscopic examination of intact human gastrointestinal mucosa ex vivo. Clin. Gastroenterol. Hepatol. 6, 95-101 (2008).

10. Glaser, A. K. et al. Light-sheet microscopy for slide-free non-destructive pathology of large clinical specimens. Nat. Biomed. Eng. 1, 1-10 (2017).

11. Poola, P. K., Afzal, M. I., Yoo, Y., Kim, K. H. \& Chung, E. Light sheet microscopy for histopathology applications. Biomed. Eng. Lett. 9, 279-291 (2019).

12. Orringer, D. A. et al. Rapid intraoperative histology of unprocessed surgical specimens via fibre-laser-based stimulated Raman scattering microscopy. Nat. Biomed. Eng. 1, 1-13 (2017).

13. Lu, F.-K. et al. Label-free neurosurgical pathology with stimulated Raman imaging. Cancer Res. 76, 3451 (2016).

14. Wong, T. T. W. et al. Fast label-free multilayered histology-like imaging of human breast cancer by photoacoustic microscopy. Sci. Adv. 3, e1602168 (2017)

15. Bell, K. et al. Reflection-mode virtual histology using photoacoustic remote sensing microscopy. Sci. Rep. 10, 19121 (2020).

16. Abbasi, S. et al. All-optical reflection-mode microscopic histology of unstained human tissues. Sci. Rep. 9, 1-11 (2019).

17. Yao, D.-K., Maslov, K., Shung, K. K., Zhou, Q. \& Wang, L. V. In vivo label-free photoacoustic microscopy of cell nuclei by excitation of DNA and RNA. Opt. Lett. 35, 4139-4141 (2010).

18. Ecclestone, B. R. et al. Histopathology for Mohs micrographic surgery with photoacoustic remote sensing microscopy. Biomed. Opt. Express 12, 654 (2021).

19. Ecclestone, B. R. et al. Improving maximal safe brain tumor resection with photoacoustic remote sensing microscopy. Sci. Rep. 10, $17211(2020)$.

20. Vakoc, B. J., Fukumura, D., Jain, R. K. \& Bouma, B. E. Cancer imaging by optical coherence tomography: preclinical progress and clinical Potential. Nat. Rev. Cancer 12, 363-368 (2012).

21. Nolan, R. M. et al. Intraoperative optical coherence tomography for assessing human lymph nodes for metastatic cancer. $B M C$ Cancer 16, 144 (2016).

22. Huang, Y. et al. Optical coherence tomography detects necrotic regions and volumetrically quantifies multicellular tumor spheroids. Cancer Res. 77, 6011-6020 (2017).

23. Kut, C. et al. Detection of human brain cancer infiltration ex vivo and in vivo using quantitative optical coherence tomography. Sci. Transl. Med. 7, 292ra100 (2015).

24. Adler, D. C. et al. Three-dimensional endomicroscopy using optical coherence tomography. Nat. Photonics 1, 709-716 (2007).

25. Marchand, P. J. et al. Visible spectrum extended-focus optical coherence microscopy for label-free sub-cellular tomography. Biomed. Opt. Express 8, 3343-3359 (2017).

26. Gambichler, T. et al. Comparison of histometric data obtained by optical coherence tomography and routine histology. J. Biomed. Opt. 10, 044008 (2005).

27. Beard, P. Biomedical photoacoustic imaging. Interface Focus 1, 602-631 (2011).

28. Kedarisetti, P., Haven, N. J. M., Restall, B. S., Martell, M. T. \& Zemp, R. J. Label-free lipid contrast imaging using non-contact near-infrared photoacoustic remote sensing microscopy. Opt. Lett. 45, 4559-4562 (2020). 
29. Liu, W. \& Yao, J. Photoacoustic microscopy: Principles and biomedical applications. Biomed. Eng. Lett. 8, 203-213 (2018).

30. Hajireza, P., Shi, W., Bell, K., Paproski, R. J. \& Zemp, R. J. Non-interferometric photoacoustic remote sensing microscopy. Light Sci. Appl. 6, e16278-e16278 (2017).

31. Wang, P., Wang, P., Wang, H.-W. \& Cheng, J.-X. Mapping lipid and collagen by multispectral photoacoustic imaging of chemical bond vibration. J. Biomed. Opt. 17, 096010 (2012).

32. Reza, P. H., Bell, K., Shi, W., Shapiro, J. \& Zemp, R. J. Deep non-contact photoacoustic initial pressure imaging. Optica 5, 814-820 (2018).

33. Ecclestone, B. et al. Towards virtual biopsies of gastrointestinal tissues using photoacoustic remote sensing microscopy. Quant. Imaging Med. Surg. 11, 1070-1077 (2020).

34. Ecclestone, B., Dinakaran, D. \& Reza, P. H. Single acquisition label-free histology-like imaging with dual contrast photoacoustic remote sensing microscopy. J. Biomed. Opt 26, 056007 (2021).

35. Hosseinaee, Z. et al. Functional and structural ophthalmic imaging using noncontact multimodal photoacoustic remote sensing microscopy and optical coherence tomography. Sci Rep 11, 11466 (2021).

36. Martell, M., Haven, N. J. M. \& Zemp, R. J. Multimodal imaging with spectral-domain optical coherence tomography and photoacoustic remote sensing microscopy. Opt. Lett. 45, 4859-4862 (2020).

37. Martell, M., Haven, N. J. M., Restall, B. S., Kedarisetti, P. \& Zemp, R. J. Comprehensive evaluation of ex vivo and in vivo tissues with ultraviolet photoacoustic remote sensing virtual histology and spectral-domain optical coherence tomography, in Proceedings Volume 11642: Photons Plus Ultrasound: Imaging and Sensing, 116422W (2021). https://doi.org/10.1117/12.2578954.

38. Schindelin, J. et al. Fiji: An open-source platform for biological-image analysis. Nat. Methods 9, 676-682 (2012).

39. Wang, J. \& Xu, Y. Boppart. Review of optical coherence tomography in oncology. J. Biomed. Opt 22, 121711 (2017).

40. Ha, R. et al. Optical coherence tomography: A novel imaging method for post-lumpectomy breast margin assessment-a multireader study. Acad. Radiol. 25, 279-287 (2018).

41. Yao, X. et al. Visualization and tissue classification of human breast cancer images using ultrahigh-resolution OCT. Lasers Surg. Med. 49, 258-269 (2017).

42. Bell, K. L., Hajireza, P. \& Zemp, R. J. Coherence-gated photoacoustic remote sensing microscopy. Opt. Express 26, 23689-23704 (2018).

43. Abbasi, S. et al. Chromophore selective multi-wavelength photoacoustic remote sensing of unstained human tissues. Biomed. Opt. Express 10, 5461-5469 (2019).

\section{Acknowledgements}

The authors, thank Jean Flanagan at the University of Waterloo animal facility for her work in procuring Rattus tumor tissue specimens. The authors thank the following sources for funding used during this project. Natural Sciences and Engineering Research Council of Canada (DGECR-2019-00143, RGPIN2019-06134); Canada Foundation for Innovation (JELF \#38000); Mitacs Accelerate (IT13594); University of Waterloo Startup funds; Centre for Bioengineering and Biotechnology (CBB Seed fund); illumiSonics Inc (SRA \#083181); New frontiers in research fund-exploration (NFRFE-2019-01012).

\section{Author contributions}

B.R.E., Z.H., N.A., and K.B constructed the PARS and OCT system, and executed imaging experiments. B.R.E. wrote the main manuscript text and prepared the figures. D.D., and J.R.M., selected and processed clinical samples, and provided consultation in writing the manuscript. P.H.R., conceived the project and acted as the primary investigator.

\section{Competing interests}

Authors B.R. Ecclestone, K. Bell, D. Dinakaran, J.R. Mackey and P.H. Reza have financial interests in illumiSonics Inc. IllumiSonics partially supported this work. Authors Z. Hosseinaee and N. Abbasi declare no conflicts of interest.

\section{Additional information}

Correspondence and requests for materials should be addressed to P.H.R.

Reprints and permissions information is available at www.nature.com/reprints.

Publisher's note Springer Nature remains neutral with regard to jurisdictional claims in published maps and institutional affiliations.

(c) (1) Open Access This article is licensed under a Creative Commons Attribution 4.0 International License, which permits use, sharing, adaptation, distribution and reproduction in any medium or format, as long as you give appropriate credit to the original author(s) and the source, provide a link to the Creative Commons licence, and indicate if changes were made. The images or other third party material in this article are included in the article's Creative Commons licence, unless indicated otherwise in a credit line to the material. If material is not included in the article's Creative Commons licence and your intended use is not permitted by statutory regulation or exceeds the permitted use, you will need to obtain permission directly from the copyright holder. To view a copy of this licence, visit http://creativecommons.org/licenses/by/4.0/.

(C) The Author(s) 2021 